\title{
The contribution continuum revisited
}

\author{
Daniel M. Ladik and David W. Stewart
}

The (most) common mistake is not to "tell a story," but only assemble different related parts. "Telling a good story" means to critically analyze what has been done before and demonstrate convincingly why something is changing. A significant contribution to knowledge does not happen in isolation and needs to be contextualized to the current situation.

Publishing in academic journals is challenging, especially for doctoral students and young assistant professors. Despite being understood that one needs to make a contribution in order to reach final publication, making the contribution is easier conceptualized then operationalized. Specifically, for this reason, we created "The Contribution Continuum" (Ladik and Stewart 2008), asking editors their thoughts on how to make a contribution. Briefly, we defined "a contribution is made when a manuscript clearly adds, embellishes, or creates something beyond what is already known" (Ladik and Stewart 2008, p. 157).

Given the focus of this volume is on helping young scholars to develop their research, we wanted to revisit the spirit of "The Contribution Continuum" and this conversation. This time, however, we did not ask editors their thoughts on what is a contribution. By contrast, the research presented here asked reviewers:

In 150 words or less, what is the most common mistake you see when author(s) attempt to make a contribution to knowledge?

The results presented here are not from a random set of reviewers. Instead, we asked the strongest, most experienced reviewers their thoughts on common pitfalls in the manuscript creation process. With this objective, we first approached editors from the 39 SSCI marketing and related journals describing this project and then asked for a list of their strongest reviewers such as those who had won awards for their efforts, as well as reviewers who earned their way to associate or area editor positions. In total, we received 249 nominations from the editors, and we are thankful to the 104 reviewers who responded to our request for this study.

The direct question listed above produced just over 10,000 words detailing the common challenges they experience in the review process. After close analysis, we felt the four most common categories of mistakes were, authors (1) who submit grammatically unapproachable manuscripts reducing the contribution's clarity, (2) who argue that "a gap" justifies contribution, (3) who fail to position their research into an ongoing conversation in the literature, and (4) who overlook to whom (that is, which constituents) are the intended audience of their research. For each of these pitfalls, we outline the hazards using direct quotes from the reviewers. With each 
theme, we also provide recommendations on how to avoid the particular publication mistake highlighted. This research concludes with a rubric highlighting two or three pitfalls for each stage of the manuscript creation and submission process.

\section{Grammatically unapproachable manuscripts}

The most common mistake is submitting a paper that is poorly written. If it is not well written it doesn't make any difference what the content is because you cannot get to the content.

The old adage "you never get a second chance to make a first impression" rings true in the manuscript creation and contribution making process. The last thing an author wishes to do is imbue an unfavorable reaction at the outset of their contribution argument leading to reviewer comments like "a premature and sloppy initial" submission or (if) "the paper is not well-written, it makes it hard to understand the contribution." The Introduction is where scholars make the case that their research does have a contribution and is worthy of being part of the ongoing conversation in the literature. From the Introduction, the manuscript should logically flow as per the contribution statement and the outline set by the authors. Yet, authors could easily handicap themselves by confusing the reader with grammatically unapproachable text leading to reviewer comments such as, "I see a lot of papers being rejected partly because of exposition. Such papers might have had a chance otherwise, but they are so poorly written that reviewers do not see enough contribution to warrant a revision."

Similarly to grammatically unapproachable text, research is often submitted for review to which "ideas are not communicated in the most effective manner." Meaning, the presentation, logic and flow of a manuscript could just as easily obscure a contribution as poorly structured syntax. On this point, one reviewer stated, " $a$ major problem is lacking a consistently rigorous presentation of the research and by implication, its relevance." In an analogous comment, another reviewer opined that, "many manuscripts lack a precise writing style that guide the reader through the manuscript's logic and appropriateness."

Submissions that lack clarity create an unfortunate effect in the minds of the reviewer, meaning an unfavorable view of the strength of the overall contribution:

I think clarity is the key, and lack of clarity is the most common mistake. Authors need to be: (1) clear about the contribution (be specific about how the paper builds upon prior work), (2) clear about theory (precisely lay out the mechanisms and effects), and (3) clear about methods and analyses (others should be able to replicate method, and findings should be as plainly laid out as possible). If the authors aren't clear on any of these points, then the reviewers (and readers) won't know what to make of the work.

Keep in mind that it is not the reviewer's job to logically organize the author's intentions and to uncover a manuscript's true potential. Of course, the reviewers will be helpful and offer suggestions, but they also ultimately decide if the manuscript goes forward or is rejected. On this thought, one reviewer stated, "manuscripts are too long and generally not polished enough ('let's see what reviewers want'). Overall, the research is not mature enough to be sent out for review; consequently, the 
reviewer doesn't see how the authors can turn the manuscript within one round into a publishable paper." Another reviewer lamented, "problems appear when research rigor is compromised by a 'good enough' attitude. Often this appears in terms of a not-ready-for-primetime 'draft' of a manuscript that nonetheless is submitted, with the associated poor writing." Authors must understand that if they adopt a mindset with the expectation that the review process is to help them fix an undeveloped manuscript, they should expect that very few of their papers will be asked for a revise and resubmit recommendation from a reviewer.

To conclude this pitfall, this reviewer stated it well:

The number one bit of feedback I find myself giving in the review process involves the manuscript's lack of focus. So many papers I read fail to make the research contribution clear and unambiguous to the reader. The overall scope of the research often tries to accomplish too much, without probing the important question(s) with any meaningful depth. Related to this is imprecise use of terminology and lack of definitional structure. However, I see all of these as being related to the research program's lack of focus. A clearly defined focus and articulation of contribution are key to communicating the value of your work.

\section{Recommendations}

Fortunately, this pitfall has a few direct and straightforward practices which could alleviate reviewer comments like those highlighted above. First, use a professional proofreading service. As simple as this sounds, it is a valuable first step in the publication creation process, radically reducing any grammatically unapproachable text. Second, have a minimum of two or three friendly reviews to verify that the presentation, logic, and flow of your intended contribution argument is as sound as you believe it should be. Often, we are too close to our research and cannot see any other perspective than our own. Ask former doctoral program associates or new department colleagues for a friendly review. This is a great way to stay in touch with your doctoral program and also develop new relationships within your current department. Third, go back to the proofreading service after incorporating the friendly reviewer comments into the revised manuscript. One reviewer specifically suggested presenting your research at conferences:

Don't leave it to the review process to "discover" where you lack clarity. Find out beforehand by sharing your paper with colleagues, presenting at conferences, and so on, to broaden your perspective and to overcome your own subjectivity and biases. The research itself will benefit, as well as your ability to communicate that research to others.

Our final reviewer comment summarizes this theme well:

In my reviews I always recommend the submitters read chapters 1, 2, and 3 of William Zinsser's On Writing Well. One of my favorite quotes: "I write in order to understand my own ideas." When something is as poorly written as most of what I read on first submission it is evidence that the writer does not understand his or her own ideas.

\section{Arguing for "a gap" in the literature}

An identified research gap does not equal a contribution to knowledge. Oftentimes authors 
fail to move beyond gap identification and flesh out why the area is worthy of academic study. For example, how does the research gap add to theory and practice?

Simply put, "a gap" in and of itself, is not a contribution and many reviewers listed this pitfall as one of the most common mistakes in the manuscript creation process. Specifically:

Believing any "gap in knowledge" needs to be filled.

Often, authors think of their contribution in terms of "filling a gap" in the literature, and they are motivated to make this gap seem as large as possible.

To justify a research topic simply because there is no research to date yet.

Often authors confuse contribution with mere newness ("nobody yet studied this").

They argue "there is a lack" without convincingly discussing why filling it is relevant.

They focus on "gaps" in the literature versus why those gaps are important and to whom.

Focusing on novelty but forgetting about relevance. Yes, it has not been tested previously, but there might be a reason for this?

Compared to other disciplines, such as economics or psychology, the marketing literature is quite young. In other words, it is just as easy to argue that the marketing literature is nothing but gaps as it is to argue that any one gap could be a significant contribution. Metaphorically speaking, when we look out into the night sky, we can see nothing but stars. In reality, there is significantly more dark space between those stars than we can easily comprehend. By focusing just on one gap between stars, we might lose the bigger picture and miss an entire constellation. One reviewer touched on this point well, stating:

[A]uthors are defining their contribution in a narrow and specific way that suits their data or approach. The review of the existing literature is often not comprehensive enough, and the bigger picture is missed. Authors also see an area that has not been researched as a white space where the contribution is obvious ("first to study this"), but the relevance of the research question is ignored (maybe that is why others have not looked at it).

Reviewers identified two specific author pitfalls when arguing that "a gap" is a contribution. First, authors assume that "a gap" also equates to "being interesting." One reviewer delineated this point perfectly, "many authors still think that researching something that has not been researched before, is in itself a contribution. However, if a certain relationship has not been researched before, it might simply be because there's nothing interesting to research there." The second "gap" mistake is when authors confuse a theoretical gap with a contextual or constituents' gap. For instance, "too often authors confuse a different context for a contribution. The author says 'it hasn't been done in country $X$ or discipline $Y$ ' when that is, without justification, 
insufficient for a contribution to knowledge. Why would we expect it to be different in the different context?" Another reviewer lamented that authors, "focus too much on the theoretical 'gap' without seeing if that gap is there for a reason (that is, no point in exploring) or if filling the gap is actually consequential for some stakeholders."

\title{
Recommendations
}

Our strongest recommendation is for authors to not only remove "gap" verbiage from their manuscripts, but also to remove "gap" thinking from their mindset. When trying to make a contribution in a literature that is nothing but gaps, authors could be handicapping their contribution argument from the outset, therefore thinking myopically. The concern here is that even a well-written and methodologically rigorous manuscript, but which lacks an interesting question, still has the potential to be rejected, especially from the top publications, as this reviewer suggested well:

\begin{abstract}
A junior researcher may often err on the side of conservatism and in wanting to secure a safe and possibly quick publication will limits his or her research imagination to the confirm or disconfirm empirical findings. Work produced may be technically sound but the value of contribution points to our discipline, modest. They may take comfort in trialed and tested gap-spotting techniques to justify their work, and in doing this end up producing work that while rigorous, is not always interesting.
\end{abstract}

In closing, if an author focuses more on the "gap" and not on the literature around their contribution, it's too easy for a reviewer to dismiss the research as "minor" or "incremental." Foreshadowing towards the next theme, authors should focus less on the theoretical "gap" and more on how to position this contribution within the ongoing conversation in the literature.

To conclude this theme, this reviewer summarized this theme well:

Authors say - in so many words - that they shed light on something "new" or not well understood but don't tell us what they then "see" or what we're supposed to learn; or they identify a gap but don't tell us why it is important to learn something new about the "gap" or don't bother to tell us how they fill the gap and why we should be interested. But most often the problem is that authors do not identify a clear and coherent context of theoretical contribution at all - they point out all kinds of gaps, problems, aims, and objectives but don't explain what their study actually shows and how their findings complement, correct and extend some existing stream of literature/body of theoretical knowledge.

\section{Where is the conversation?}

They do not explicitly articulate what has been done in the literature, what the article adds and why that is relevant. Hence very often the main issue is that the contribution (which may be present) is not sufficiently well spelled out. Most research would benefit from making a stronger case for how the article changes/deepens our insights into a generalizable, theoretically sound phenomenon.

While arguing for "a gap" in the literature may be the most common pitfall itemized by the reviewers, failing to position their contribution within an ongoing conversation in the literature is the most devastating mistake authors make in the 
manuscript creation process. Not clearly articulating what your research has added beyond what is already known is setting oneself up for failure in the review process. Not surprisingly, many reviewers strongly noted this theme stating, "a frequent mistake is that authors do not make the CONSEQUENCES of their study explicit. What exactly follows from it, and which value does this piece of knowledge add for what reasons?"

Related to not articulating the contribution well, reviewers commented that authors also do not position their contribution well vis-à-vis what has preceded their submitted research and how it will change the conversation going forward, explicitly, "they don't position their work clearly. Without a clear positioning (that is, what work does this build on? - what do we already know?), it is hard for the review team to assess the magnitude of the contribution." Another reviewer recommended the importance of accomplishing this goal in the Introduction, detailing, "not being clear and straightforward about the (intended) contribution over the existing research in the Introduction (first 1-3 pages). (Realistic) Positioning is critical at the beginning so as to steer how a reviewer reads and evaluates the study."

\section{Recommendations}

Our definition of what is a contribution (Ladik and Stewart 2008) is very specific. We view the literature as an ongoing conversation and contribution occurs when an author adds something beyond what is already known to that conversation. The implication of this is that the ongoing conversation in the literature will change as a result of your research. If another researcher attempts to join this conversation after your research is published in the literature, your research should be referenced as part of their contribution argument.

When talking to young scholars about making a contribution to the literature, perhaps an apt analogy is that of a house party. We have all experienced this phenomenon; when attending a house party, for some reason or another, everyone ends up in the kitchen. Let's pretend that you were a little late to the party, but you wanted to join this lively conversation in the kitchen which happens to presently focus on the English Premier League. After listening to the ongoing conversation for a while that focused on arguments pertaining to which teams will finish in the Top 4 and in what order, a short lull in the conversation occurs. Recognizing this opportunity to join the conversation, you interject, "Did you see what happened in the Formula 1 race this weekend in Monaco? What a crash!"

While the Monaco comment was stated with much enthusiasm, it did not add anything to the ongoing conversation about the Top 4 teams in the English Premier League. The comment was not positioned well versus the comments on the teams who are in contention for those Top 4 slots. The comment did not add anything beyond what was already known, and the conversation in the kitchen did not change as a result of your thoughts. Maybe there is an active Formula 1 conversation in the garage or out on the lanai one could join. In the kitchen, however, the Monaco comment did not change the conversation, and therefore, you did not make a meaningful contribution.

To avoid this particular pitfall, we recommend that authors clearly outline how the research adds something beyond what is already known and what could change in the 
literature. Many authors treat the Introduction and the Literature Review sections as "history lessons." The reviewer does not need a historical account in summary form of previously published literature. In the top journals, many of the reviewers selected by the editor will most probably be the references the author summarizes in their history lesson. Furthermore, when authors present a history lesson, they invariably struggle with developing strong hypotheses because they are not specific to what could change in the ongoing conversation in the literature as a result of their analysis.

As opposed to a "history lesson", authors should focus on how their research "fits" into the conversation that preceded it. Illustratively speaking, the following shows an example of how an author could position their research:

- Some author publishes Paper A which creates the construct of Subject X and found an effect.

- Another author publishes Paper B on Subject X found this effect again and added another theory to extend its robustness.

- A third author publishes Paper C on Subject X found this effect again but outlined explicit boundary conditions for the effect.

- You then submit your research, Paper D on Subject X for review to a journal. You clearly position Paper D versus the ongoing conversation in the literature (Papers A, B, C) and show via classic theory testing, which theories in the literature hold and which do not agree with the theory that supports Subject X. As a result, the conversation in the literature (Papers A, B, and C) changed as a consequence of Paper D. All further papers on Subject X should appropriately reference Paper D.

To conclude this pitfall, the comment below hits many of the pain points outlined by the reviewers: (1) not explicitly stating their contribution, (2) arguing for some sort of " $g a p$ " as opposed to positioning a contribution within an ongoing conversation, (3) failing to state their contribution clearly in the Introduction, and (4) not exploring friendly reviews and outside opinions before journal submission to verify that the presentation, logic, and flow of a manuscript is sound:

The most common mistake I see is that authors do not explicitly state their contribution to knowledge. They suggest a topic has not been studied before, or that two concepts have not been studied together, but they never say why their research question should be answered, why it is important to theory, and what contribution it makes to the field. If they would take the time to explicitly write out their contribution - in the introduction of the paper - they would often find out that the contribution is not clear in their own minds, or that it is smaller than they thought it was. Or non-existent.

\section{Who is the intended audience?}

Casting their contribution in terms of "it's never been done before" rather than in terms of "here's how the behavior of an important constituency will change as a result of our paper."

Once the authors have established the contribution (that is, adding something beyond what is already known) and how it is positioned within the ongoing 
conversation in the literature, authors must be mindful of who will be impacted by this new information. The opening reviewer comment uses the word "constituency," and we cannot think of stronger terminology to describe this pitfall. Every published paper has an intended audience and some authors, "overlook to whom (that is, which constituents) are the intended audience of their research."

Many papers have an academic audience and authors should be clear on how the conversation will change among this community. Please note, some academics are looking for a theoretical contribution, but it may also be the case that an academic audience could be looking for a change in teaching practice or novel ideas on running a business school. Some reviewers complained about overlooking non-academic audiences with overly complex methodology, stating "by design, many projects have no chance of making a compelling contribution to non-academic stakeholders." Another reviewer pointed directly to a managerial audience, stating, "not adequately explaining 'why' addressing the gap is important for knowledge development and/or managerial practice." Lawmakers, both on the local level or in Washington D.C., could be another vitally important constituency impacted by your research. Society at large is also another significant constituency which could be impacted by academic research.

\section{Recommendations}

Not only should the contribution be stated clearly in the Introduction of the manuscript, but the authors should also be explicit on who will be impacted by the results. We suggest this is an issue authors discuss meticulously with their friendly reviewers. Some of our top journals are general, like the Journal of Marketing, meaning the onus is on the authors to be specific on audience impact. Other journals have certain constituencies preordained as per the mission of the publication such as the Journal of Retailing, the Journal of Public Policy \& Marketing or Journal of Advertising. We recommend not only to review the target outlet's mission statement, but also review three or four of the most recent letters from the editor which outline the current editor's mindset. Invariably, the editors will detail contribution areas and constituencies for the publication.

To conclude the four pitfalls, the following reviewer said it well:

Contributions to knowledge are relatively subjective and do entail the question of "contribution to whom." As a researcher interested in the intersection of consumer wellbeing and public policy, with a focus on the broad potential for substantive impact for society at large, I have become concerned with papers that seem to be so focused on finding a unique "surprise" that the specific research procedures, methods, and analyses are designed to only deliver the extremely novel finding. This sometimes seems to lead to extremely unusual procedures, methods, and contexts associated with the desire for the "surprise" finding. If this contribution to knowledge is solely focused on the theoretical domain, this may be very reasonable. However, if the implications for the substantive domain are positioned as of interest, the contribution seems limited, and, in a worst-case scenario, potentially misleading. If there are reported implications to applied marketers or society at large, generalizability and replicability of findings become important. Clarifying the contribution to what type of knowledge and for whom the knowledge is intended is critical. 


\section{Conclusion: What is the story?}

The most common mistake here is the absence of an overarching theoretically anchored conceptual story in the study. This absence of such a story limits the potential of the study to make a substantive contribution to existing knowledge in terms of theory development and the advancement of management practice in the field.

The goal of this chapter, as well as the other chapters in this volume, is to help young scholars develop their research and make a strong contribution. With this objective, we asked some of the strongest reviewers in our discipline what are the most common manuscript mistakes, and they expressed, authors (1) who submit grammatically unapproachable manuscripts reducing the contribution's clarity, (2) who argue that "a gap" justifies contribution, (3) who fail to position their research into an ongoing conversation in the literature, and (4) who overlook to whom (that is, which constituents) are the intended audience of their research.

The opening quote of this chapter and the one that initiates the Conclusion section highlights the final pitfall we would like to stress to developing scholars; "The most common mistake I see is when authors do not know how to turn significant research findings into a forceful, compelling narrative, thereby making it unclear what the contribution is" (emphasis added). Although each manuscript has a set of distinct sections that are in a common order, it does not follow that a collection of disjointed sections that do not mesh well together creates a contribution. This is especially apparent when the manuscript has multiple authors who are each responsible for their respective section and do not review for article cohesiveness. Moreover, the lack of "a forceful compelling narrative" leads to exploring smaller or minor research questions as opposed to something more substantial:

When I review, I am having to use too many times a concluding sentence of the kind "In sum, I suggest taking a step back and reconsider the research question." As Eitan Muller told the IJRM ERB few years ago, we focus on providing perfect answers to small questions instead of providing good answers to big questions.

What is missing from many manuscripts is a what one reviewer called a "strong narrative arch", detailing:

Another, shortcoming I often encounter is the lack of a strong narrative arch. What I mean by that is that often authors conduct a series of studies and then superficially combine them into the manuscript without really focusing on what is the story here and thereby what is the contribution. Just because the results are significant does not mean that the contribution is worthwhile.

Each section of the manuscript should logically lead to the next and the storyline should be consistent throughout. The Abstract should match the Introduction and Discussion sections. The Introduction states the contribution and sets the outline for the manuscript. The Literature Review succinctly positions the conversation towards the research Hypotheses. The Method is appropriate for the research questions argued for in the Introduction. The Results are not overstated and the Discussion not only matches the Introduction but also does not make conclusions which are not supported 
by the data. Research is, "about using evidence, theory, and logic to weave a 'true' story; when that story is less than compelling, the ultimate result is research that lacks impact and fails to advance domain knowledge (and thus often fails to publish well)." The most common word a reviewer will use when "the story" or "narrative arch" is lacking, is "disconnect" as the presentation, logic, and flow of a manuscript is disjointed therefore clouding the contribution argument.

In closing, we are indebted to the 104 expert reviewers who took the time to provide the treasure trove of excellent comments for the manuscript constructionand contribution-making process. We hope these words of wisdom will echo in your thoughts when working on your research projects. That said, not all of their comments fit neatly within the four dominant themes outlined in this chapter. At the same time, we did not want these valuable comments to fall by the wayside and instead, used them to create The Master Mistake Rubric which is included in the Appendix. The rubric highlights, section by section, common pitfalls in the manuscript creation process and we hope you keep this document handy, therefore avoiding these surefire mistakes. Best of luck with your research!

\section{Reference}

Ladik, Daniel M. and David W. Stewart (2008), "The Contribution Continuum," Journal of the Academy of Marketing Science, 36, 157-65. 


\section{Appendix: The Master Mistake Rubric: sure-fire strategies to guarantee article rejection}

The most common mistake I see is that authors often fail to remember that editors and associate editors are tasked with publishing papers that are Accurate, Impactful, and Rigorous (AIR). It's not enough to have one of these things without the others. For example, many authors work very hard to produce air-tight, rigorous research, and then they may be surprised that it gets rejected because it doesn't do enough to move the discipline forward. Others may have a "big idea" that has the potential to drive future research but falls short because the paper lacks rigor. Underneath both of these issues is the pitfall where authors often look to prior research published in a journal and assume that the contribution level and rigor required to publish an article in the past is sufficient today. We must move forward as a discipline and, if we're successful, requirements on all three elements of AIR must increase over time.

\section{Before submission}

- "Misalignment with the journal, not speaking to the audience of the journal."

- "Another mistake is that the contribution does not fit the audience of the journal. For instance, authors provide a substantial contribution, but the journal is looking for theoretical contributions."

- "Poor writing. Problems with the organization of the paper, problems with clarity, grammar issues."

- "They choose something that is too narrow. That is, the contribution is too narrow to be meaningful for any stakeholder (researchers, marketing managers, public-policy makers, and so on.)"

- "The most common mistake I see is that authors don't really position papers for the journals they are targeting, so that I am left wondering whether they had even read a single paper in the journal the paper has been submitted to."

Title and abstract

- "Other mistakes that lead to a rather negative impression straight from the start are an unbalanced abstract (often emphasizing the study's background rather than findings or value of them) or an abstract appearing different from the actual paper, and when the introduction is sloppily written or feels too broad or beside what the title or abstract presented."

- "Overpromising in the title or the abstract. This leaves the reader feeling underwhelmed at best and misled at worst."

- "The abstract often summarizes the paper but doesn't necessarily highlight what is new for different audiences and sets of literature. This is assuming there is a contribution in the first place, of course."

\section{Introduction}

- "The first is not clearly communicating, early in the paper (e.g., the introduction) exactly what the contribution of the paper is and how it moves us beyond what is currently known. I tell my doctoral students that they 'must' be able to do this 
clearly and early as this is what will ultimately catch the reader's attention and cause them to read the paper with interest."

- "Typically, the introduction does not effectively establish the study's importance or its contribution to the extant literature."

- "Young scholars frequently do not adequately show how their work augments or extends previous work (by using, for example, a literature table listing existing and intended new contributions)."

- "Taking four pages to state the objectives of the paper. These should be clear after a paragraph or two. If it is hard to explain the contribution, odds are there is little."

\section{Literature review}

- "A key mistake is not anchoring the research in a strong theory. This is evident in the literature review where the discussion does not identify the key issues that need closer examination."

- "Presenting the literature review as a set offacts instead of offering implications. The point of the review is to indicate why and how the paper differs (or to set up the design of the study)."

- "Oftentimes, the conceptual framework is underdeveloped, overly brief, and based on implied or unsubstantiated opinion. Other problems with the conceptual framework include blending several theoretical foundations together without establishing their compatibility; and, introducing theories from different disciplines without establishing their importance, relative to related theories in marketing, or adequately explaining them."

- "Most researchers don't ever identify a good set of research questions to guide their project. They ask relatively mundane questions based on past papers they have read, that are not very interesting or novel."

- "The paper is not well positioned in the literature (how does it add to the conversation?)."

- "The article ignores past research (claiming a contribution when there is not)."

- "Not fully accounting for the existing literature. In order to contribute to literature, the author needs to start from a foundation of what is already known."

- "Not offering meaningful insight in conceptual development (citing facts, not offering analysis/synthesis)."

- "Not knowing previous literature so arguing something is a significant contribution even if it's minor or has already been shown."

Hypotheses

- "Common difficulties with the research design concern the measurement scales. Oftentimes, scales are created or adapted without adequately establishing their appropriateness for examining the research hypotheses/ questions."

- "Every hypothesis should have a rigorous rationale behind it, but in my 
personal opinion the most interesting questions are: (1) those where the hypothesis can go multiple ways (e.g., the DV goes up or down) and there are theoretically different explanations for it or (2) those where authors aim to explore an interesting phenomenon that we see happening in the managerial world."

- "A related problem is that oftentimes, it seems as if the author is developing hypotheses post hoc after the data analysis reveals some significant results, that were not expected or part of the original theoretical investigation."

- "By this I mean that authors often describe their hypothesis but fail to connect the hypothesis to prior literature and/or fail to explain how the differences between prior literature and the current work are meaningful/important."

Method

- "Not considering if their research method best answers the research question posed. Specifically, given the research question, what makes their methodology, data, institutional context the best-in-class in terms of answering the question?"

- "The article includes moderators or mediators without any theoretical basis."

- "Authors find relationships between variables in data sets and attempt to position the paper as if the hypotheses were a priori."

- "The paper mixes 'theories' to propose paths in a theoretical model."

- "The constructs and variables are not (well) defined."

- "Flawed methods. Incomplete reporting, confounded study designs, inadequate methods, procedural issues in the study."

- "Not connecting the conceptual argument to the methodology/analysis that is chosen."

Discussion

- "An additional clear mistake is a section with unclear theoretical and managerial implications."

- "The discussion section oftentimes does not identify new insights gained from the study findings or explain how they add meaningfully to the literature."

- "Not considering implications beyond theory (that is, public policy, managerial implications)."

- "Papers often lack implications. If there are no policy relevant variables, it is often unclear what any decision maker should do with the findings. Conjecture is not sufficient."

- "Related to this is an inability to interpret what the research has found and impart meaning to it. Often the managerial implications are forced or nonexistent."

- "Authors fail to return to develop the theoretical postulates offered in the literature review in their discussion."

- "Authors fail to distinguish sampling boundaries from theoretical 'limitations' of the research." 